\title{
Dietary composition is associated with one-carbon metabolites and B-vitamin status in patients with stable angina - a cross-sectional study
}

\author{
Vegard Lysne $^{1}$, Anthea van Parys ${ }^{1}$, Thomas Olsen ${ }^{2}$, Ingvild Marienborg ${ }^{1}$, \\ Johnny Laupsa-Borge ${ }^{1}$, Teresa Risan Haugsgjerd ${ }^{3}$, Per Magne Ueland ${ }^{4}$, Jutta Dierkes ${ }^{5,6}$ and \\ Ottar Nygård ${ }^{1,7}$ \\ ${ }^{1}$ Centre for Nutrition, Department of Clinical Science, University of Bergen, Bergen, Norway, \\ ${ }^{2}$ Department of Nutrition, University of Oslo, Oslo, Norway, \\ ${ }^{3}$ Department of Global Health, University of Bergen, Bergen, Norway, \\ ${ }^{4}$ Bevital AS, Bergen, Norway, \\ ${ }^{5}$ Centre for Nutrition, Department of Clinical Medicine, University of Bergen, Bergen, Norway, \\ ${ }^{6}$ Laboratory Medicine and Pathology, Haukeland University Hospital, Bergen, Norway and \\ ${ }^{7}$ Department of Heart Disease, Haukeland University Hospital, Bergen, Norway
}

\begin{abstract}
Introduction: Peroxisome Proliferator-Activated Receptor $\alpha(\operatorname{PPAR} \alpha)$ is a known regulator of lipid and energy metabolism. In animal experiments of PPAR $\alpha$ activation, we have reported on altered plasma concentration of metabolites related to one-carbon metabolism and B-vitamin status. Several of these metabolites, e.g. homocysteine and dimethylglycine, have been associated with increased cardiovascular disease risk. Diet can influence one-carbon metabolism, and this may partly be mediated through altered PPAR $\alpha$ activity as dietary fatty acids can activate PPAR $\alpha$. As diet is a modifiable life style factor, the aim of this investigation is to explore potential associations between dietary composition and plasma concentration of one-carbon metabolites and markers of B-vitamin status.
\end{abstract}

Materials and methods: The study population consisted of 1977 patients with stable angina (geometric mean age 61 years, $80 \%$ male) from the Western Norway B-vitamin Intervention Trial (WENBIT), who completed a self-administered food frequency questionnaire at baseline. Data on outcome variables were obtained from the baseline blood samples ( $35 \%$ fasting). Outcome variables of interest were metabolites related to one-carbon metabolism and B-vitamin status. Cross-sectional associations between protein, carbohydrate and fat intake with the outcome metabolites were analysed with linear regression, adjusted for age, sex, fasting status, BMI and reported energy intake. Estimates are given as \% change in the outcome variable per isoenergetic increment of 1 energy $\%(\mathrm{E} \%)$ in the exposure nutrient.

Results: The average distribution [95\% prediction interval] of energy intake ( $\mathrm{E} \%$ ) in the population was $48.5(37.1,63.4)$ from carbohydrate, $31.4(21.9,44.9)$ from fat and $16.5(12.2,22.3)$ from protein.

The strongest associations were seen for increasing protein intake, while the associations with fat and carbohydrate intake were weaker. The most pronounced associations ( $\%$ change $[95 \%$ confidence interval]) with increasing protein intake were higher cobalamin $(2.9[2.1,3.7])$, PLP $(2.7[1.7,3.7])$ and riboflavin $(2.3[1.0,3.6])$, and lower tHcy $(-1.4[-1.9,-0.9])$ and MMA (-1.3 [-1.9, -0.7]).

Discussion: The current observations indicate that dietary macronutrient composition may influence plasma concentration of onecarbon metabolites and markers of B-vitamin status. The strongest associations are observed with increasing protein intake, but elucidating the importance of the nutrient substituted remains. Whether the observed associations are due to macronutrients per se, or reflect differences in food choices/nutrient intakes, or effects on physiological factors, i.e. inflammation or PPAR $\alpha$ activation, is not known.

\section{Conflict of Interest}

There is no conflict of interest 